\title{
Brasagem da zircônia metalizada com titânio à liga Ti-6Al-4V
}

\section{(Brazing of metalized zirconia with titanium to Ti-6Al-4V alloy)}

\author{
J.S.Pimental , A.J.A.Buschinelli ${ }^{2}$ R. M.do Nascimento ${ }^{3}$,A.E. Martinelli ${ }^{3}$, U.Reisgen ${ }^{4}$ \\ ${ }^{I}$ Instituto Federal de Santa Catarina, Campus Avançado Xanxerê, Xanxerê, SC, Brasil 89820-000 \\ ${ }^{2}$ Labsolda, Departamento de Engenharia Mecânica, Universidade Federal de Santa Catarina, campus Trindade, \\ CP 476, Florianópolis, SC, Brasil 88040-970 \\ ${ }^{3}$ Departamento de Engenharia de Materiais,Universidade Federal do Rio Grande do Norte, campus Lagoa \\ Nova, CP 1524, Natal, RN, Brasil 59072-970 \\ ${ }^{4}$ Welding and Joining Institute, RWTH Aachen University ISF, Germany \\ jean.pimenta@ifsc.edu.br
}

\begin{abstract}
Resumo
Zircônia tetragonal estabilizada com ítria foi mecanicamente metalizada com titânio e a condição de molhamento avaliada com as ligas convencionais Ag-28Cu e Au-18Ni. Estas dissolveram o revestimento de titânio para uma completa distribuição deste metal ativo na superfície cerâmica, gerando uma liga ativa in situ e possibilitando adequadas ligações químicas ao metal base na temperatura de união. Os melhores resultados de molhamento foram selecionados para brasagem indireta em forno de alto-vácuo nas juntas $\mathrm{ZrO}_{2} / \mathrm{Ti}-6 \mathrm{Al}-4 \mathrm{~V}$. Testes de detecção de vazamento de gás hélio foram realizados na interface de união das juntas; amostras removidas na seção transversal de juntas estanques foram examinadas por técnicas de análise microestrutural. Formou-se uma camada escura adjacente à cerâmica metalizada, responsável pelo molhamento ocasionado pela liga $\mathrm{Ag}$ - $28 \mathrm{Cu}$. Entretanto, o uso da liga $\mathrm{Au}-18 \mathrm{Ni}$ resultou em precipitação de intermetálicos e microtrincamento interfacial. Perfis de microdureza através da interface resultante até onde a zircônia mostrou típico escurecimento não indicaram alternância significativa entre medições consecutivas; os resultados dos ensaios de resistência mecânica à flexão-3p foram considerados satisfatórios.
\end{abstract}

Palavras-chave: metalização mecânica, zircônia, brasagem, união cerâmica/metal.

\begin{abstract}
Yttria tetragonal zirconia polycrystal was mechanically metallized with titanium and the wetting behavior on the ceramic surface was analyzed using the conventional fillers Ag-28Cu and Au-18Ni. These alloys had dissolved the active metal coating, which acts to zirconia reduction on its surface and promoting suitable chemical bonding to the metallic member. Better wetting results were selected for indirect brazing in a high-vacuum furnace for $\mathrm{ZrO}_{2} /$ Ti-6Al-4V simple butt joints. Helium gas leak detection was made at the joints interface; samples were removed from the tight joints cross-section and examined by microstructural analysis techniques and EDX analysis. There was formation of a dark reaction layer close to the metalized ceramic surface, which was responsible for its good wetting behavior using the filler Ag-28Cu. However, the Au-18Ni filler alloy caused an excessive presence of intermetallics and interfacial microcracking. Microhardness profiles were made across the joints interface to where zirconia undergone typical darkening and there was no significant variation between consecutive measurements; performance of brazed joints was considered satisfactory by three-point flexure testing.
\end{abstract}

Keywords: mechanical metallization, zirconia, brazing, ceramic/metal joining.

\section{INTRODUÇÃO}

Os campos de aplicação das cerâmicas avançadas estão relativamente associados à crescente demanda por uniões cerâmica/metal, acarretando a produção de componentes híbridos com interfaces confiáveis e melhoradas propriedades de engenharia. Neste grupo incluem-se alguns compósitos cerâmicos, óxidos e nitretos, merecendo destaque a alumina $\left(\mathrm{Al}_{2} \mathrm{O}_{3}\right)$ e zircônia $\left(\mathrm{ZrO}_{2}\right)$ como as cerâmicas óxidas mais comumente usadas em pesquisas visando possibilidades de aplicação industrial na união com metais $[1,2]$.

A zircônia apresenta alta resistência à corrosão em elevadas temperaturas e maior resistência mecânica e tenacidade à fratura comparativamente a outras cerâmicas densas, particularmente em temperaturas abaixo de $\sim 300{ }^{\circ} \mathrm{C}$ para valores $K_{\mathrm{IC}}$ na faixa de 15-20 MPa.m ${ }^{1 / 2}$ [3]. Estas são propriedades importantes para uma extensa faixa de aplicações tecnológicas desde moldes para trefilação de arames, ferramentas de corte e usinagem, componentes em turbinas até sensores de oxigênio e células-combustível de óxido sólido $[1,4,5]$. Esta cerâmica requer uma adequada transformação de fase tetragonal para monoclínica $(t \rightarrow m)$, necessária para promover alta resistência à abrasão e melhores propriedades mecânicas. Adições de certos óxidos, tais como magnésia $(\mathrm{MgO})$ ou ítria (em geral 3,2-4,2 mol\% ou $\sim 6-8$ peso $\% \mathrm{Y}_{2} \mathrm{O}_{3}$ ) e rigoroso tratamento térmico são cruciais para sobrepujar o aumento 
de volume de 3-5\% na zircônia pura devido à transformação $t \rightarrow m[3,6]$. As tensões induzidas seriam suficientes para gerar um trincamento espontâneo na zircônia pura durante o resfriamento a partir da temperatura de sinterização [7].

$\mathrm{O}$ processo de brasagem é relativamente simples para a união entre metais ou mesmo de cerâmicas aos metais, usando-se uma liga de adição contendo um metal quimicamente ativo (Ta, $\mathrm{Nb}, \mathrm{Zr}, \mathrm{V}$, Hf e especialmente Ti). Pelo fato dos materiais cerâmicos serem de custo elevado e apresentarem dificuldades na fabricação de componentes grandes e/ou de geometria complexa, tal processo vem sendo continuamente pesquisado $[2-4,8]$. Como regra geral, um metal ativo deve reduzir quimicamente o principal óxido constituinte da cerâmica, a fim de favorecer o molhamento da liga fundida na superfície cerâmica e se obter adequadas ligações químicas entre esta e o metal; igualmente relevante é que o metal ativo deverá possuir alta afinidade química pelo oxigênio [5, 9, 10]. Entretanto, os maiores custos das ligas ativas em comparação às convencionais pode ser considerado uma desvantagem. Isto tem direcionado pesquisas alternativas, nas quais se pode incluir uma etapa prévia de metalização na cerâmica e a posterior brasagem indireta [11, 12].

Os componentes a serem unidos por brasagem deverão possuir similares coeficientes de expansão térmica linear ( $\alpha$-CTE). Por exemplo, no caso da zircônia parcialmente estabilizada (cerâmica PSZ) e titânio de pureza comercial (ASTM grau 4) os valores de $\alpha$-CTE são similares $\left(\sim 10 \times 10^{-6} /{ }^{\circ} \mathrm{C}\right.$ e $\sim 11 \times 10^{-6} /{ }^{\circ} \mathrm{C}$, respectivamente), sendo importante para minimizar as tensões residuais geradas na interface da junta no resfriamento a partir da temperatura de união $[2,3,8]$. Outra questão relevante é que a liga de adição deverá ter, necessariamente, sua temperatura liquidus maior do que a temperatura de trabalho da junta e ao mesmo tempo ser inferior às temperaturas de fusão dos componentes individuais.

Várias técnicas estão bem documentadas na literatura científica e têm estado em constante desenvolvimento para a metalização de cerâmicas, tais como plasma spray, deposição química e física de vapor (processos CVD e PVD, respectivamente) $[13,14]$. Além disso, o processo Mo-Mn é inapropriado para a metalização da zircônia porque tal cerâmica não possui uma fase vítrea intergranular em sua microestrutura [2, 15]. Não obstante, estas técnicas são desvantajosas do ponto de vista econômico.

A técnica de metalização mecânica foi originalmente proposta pelo Forschungszentrum Juelich (Alemanha) e tem sido motivo de pesquisas nos últimos anos por parte de especialistas brasileiros e alemães, sendo aplicada com sucesso em escala laboratorial na metalização de cerâmicas óxidas e não-óxidas. Uniões alumina/metal brasadas com ligas convencionais já foram obtidas em trabalhos de cooperação [11, 12, 16]. O procedimento envolve baixo custo de produção e pode ser feito à temperatura ambiente, além de não gerar resíduos nocivos ao meio. Neste estudo empregou-se esta particular técnica de metalização com titânio na obtenção de juntas estanques $\mathrm{ZrO}_{2} / \mathrm{Ti}-6 \mathrm{Al}-4 \mathrm{~V}$ com uso de ligas de adição convencionais $\mathrm{Ag}-28 \mathrm{Cu}$ e $\mathrm{Au}-$ 18Ni. Dentre os principais objetivos, incluem-se: avaliar as condições de molhamento na superfície da cerâmica metalizada e, posteriormente à brasagem indireta, caracterizar a interface de união com técnicas de análise microestrutural; além de verificar as propriedades mecânicas com testes de microdureza e resistência à flexão-3p.

\section{PROCEDIMENTO EXPERIMENTAL}

O material cerâmico utilizado foi zircônia tetragonal estabilizada com ítria (Y-TZP, Engecer Ltda., Brasil) na forma de barras $\phi 8,0 \mathrm{~mm}$. A contraparte metálica foi a liga Ti-6Al-4V (ASTM grau 5, recozida); a ferramenta cônica para metalização foi produzida a partir de uma barra de Ti comercialmente puro (ASTM grau 2). Ligas de adição convencionais (espessura $50 \mu \mathrm{m}$ ): Ag-28Cu e Au-18Ni (Brazetec, Umicore group AG \& Co. KG). Na Tabela I são dadas as principais propriedades dos materiais utilizados.

A união de zircônia a uma liga de titânio apresenta características atraentes para a obtenção de juntas em potencial por causa das mútuas aplicações dos componentes nas áreas estrutural/aeroespacial $[2,4]$. A liga Ti-6Al-4V (alpha-beta titanium alloy) é interessante devido às suas possíveis aplicações em fuselagens, partes de motores de

Tabela I - Propriedades dos materiais (dados dos fabricantes).

[Table I - Properties of the materials (manufacturer's data).]

\begin{tabular}{lcccc}
\hline \multirow{2}{*}{ Propriedades } & \multicolumn{4}{c}{ Componentes das juntas } \\
\hline Yomposição (peso\%) & $\begin{array}{c}5,0-10,0 \% \mathrm{Y}_{2} \mathrm{O}_{3}, \\
\mathrm{ZrO}_{2} \text { balaç̧o }\end{array}$ & $\begin{array}{c}\text { ASTM } \\
\text { grau } 5\end{array}$ & $\begin{array}{c}\text { 71,0-73,0\% Ag, } \\
\text { Cu balanço }\end{array}$ & $\begin{array}{c}81,5-82,5 \% \mathrm{Au}, \\
17,5-18,5 \% \mathrm{Ni}\end{array}$ \\
\hline Densidade aparente, $20{ }^{\circ} \mathrm{C}\left(\mathrm{g} / \mathrm{cm}^{3}\right)$ & $5,5-6,1$ & 4,43 & 10,0 & 15,96 \\
\hline$\alpha$-CTE $\left(\times 10^{-6} /{ }^{\circ} \mathrm{C}\right)$ & 10,4 & 9,7 & 17,8 & 14,6 \\
\hline E modulus $(\mathrm{GPa})$ & $\left(25-1000{ }^{\circ} \mathrm{C}\right)$ & $\left(20-650{ }^{\circ} \mathrm{C}\right)$ & $\left(20-400{ }^{\circ} \mathrm{C}\right)$ & $\left(20-550{ }^{\circ} \mathrm{C}\right)$ \\
\hline Dureza $(\mathrm{HRC})$ & 206 & 113,8 & 100 & - \\
\hline $\mathrm{T}_{\text {trabalho }}\left({ }^{\circ} \mathrm{C}\right)$ & 83 & 36 & - & - \\
\hline
\end{tabular}


turbinas, incluindo dispositivos cirúrgicos e implantes médicos (biocompatibilidade) [17].

A liga de adição eutética $\mathrm{Ag}$-Cu é geralmente preferida para unir cerâmicas aos metais por ser dúctil e quimicamente inerte, minimizando riscos de oxidação e formação das tensões térmicas residuais na interface de união durante o resfriamento ao final do ciclo de brasagem [2-5, 9]. Com relação à liga $\mathrm{Au}-18 \mathrm{Ni}$, esta possui excelentes condutividade térmica $(315 \mathrm{~W} / \mathrm{m} . \mathrm{K})$ e resistência à oxidação, incluindo elevada ductilidade o que também é benéfico na acomodação das tensões residuais via escoamento plástico interfacial [8].

Após o corte com disco diamantado as amostras cerâmicas foram submetidas a polimentos com suspensão de partículas de diamante 6 e 1 um, e limpas em banho de acetona no ultra-som por $10 \mathrm{~min}$. Antes de aplicar a técnica de metalização, verificou-se a rugosidade (índice $\mathrm{R}_{\mathrm{a}}$ ) na superfície cerâmica de 0,34 $\pm 0,06 \mu \mathrm{m}$. As amostras de Ti-6Al-4V foram manualmente limpas com etanol para remoção de resíduos de usinagem, seguindo banho de etanol no ultra-som.

Na Fig. 1 tem-se o esquema simplificado na técnica de metalização mecânica. A amostra cerâmica fixada na placa do torno girava no sentido anti-horário sob rotação 800 rpm, enquanto que a ferramenta metálica cônica de titânio acoplada na retificadora manual de alta rotação girava com 27.000 rpm no sentido horário ao operador; tempo de metalização $\approx 60 \mathrm{~s}$. Durante o contato, ocorre o desgaste da ferramenta paralelamente à formação do revestimento na superfície cerâmica. As variáveis de controle foram otimizadas com base no trabalho de Nascimento, quando não foi constatada uma eventual oxidação no revestimento de Ti nestas condições operacionais [11]. A configuração das juntas de topo usadas nos testes de brasagem é dada na Fig. 2, onde cada conjunto ficou submetido a uma pressão no contato $\approx 90 \mathrm{kPa}$. Esta configuração da junta de topo foi eficaz para os testes de detecção de vazamento de gás hélio a temperatura ambiente,

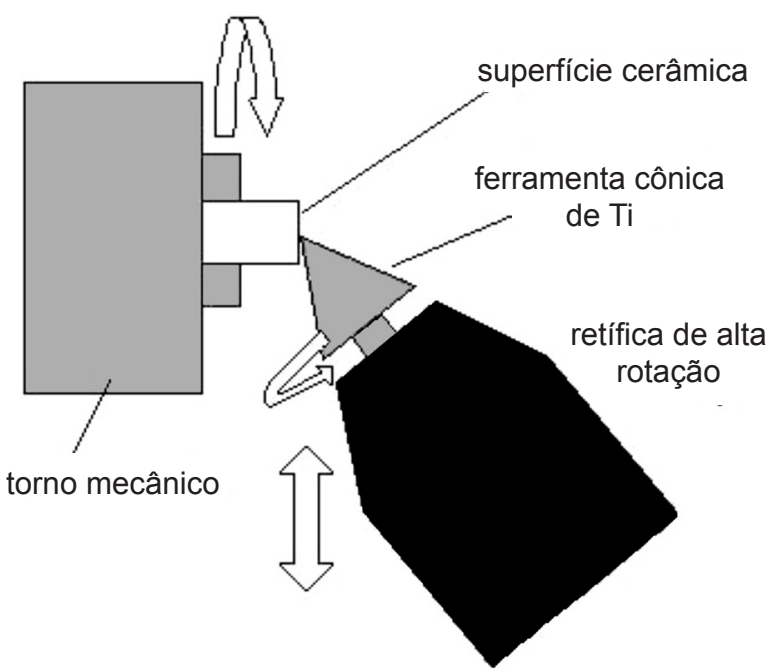

Figura 1: Ilustração esquemática da técnica de metalização mecânica (Ref. 11, adaptado).

[Figure 1: Schematic illustration of the mechanical metallization technique (Ref. 11, adapted).]
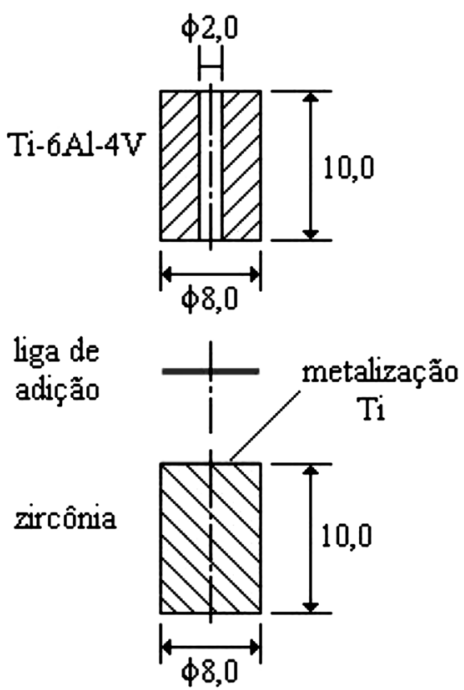

Figura 2: Configuração das juntas para os testes de brasagem (dimensão em mm).

[Figure 2: Joints configuration used for brazing tests (dimension in $\mathrm{mm}$ ).]

usando o equipamento Inficon UL200 conforme a norma DIN EN 1779.

Os testes de molhamento foram realizados num forno de alto-vácuo (Pfeiffer MOV262) na zircônia metalizada com Ti, usando as ligas de adição nos respectivos ciclos térmicos: $820{ }^{\circ} \mathrm{C} / 10$ min e $880{ }^{\circ} \mathrm{C} / 10$ min para $\mathrm{Ag}-28 \mathrm{Cu} ; 980{ }^{\circ} \mathrm{C} / 10$ min e $1050{ }^{\circ} \mathrm{C} / 10$ min para $\mathrm{Au}-18 \mathrm{Ni}$. Estas temperaturas geralmente estão $30-50{ }^{\circ} \mathrm{C}$ acima da temperatura liquidus das ligas de adição $[3,8]$. Os ângulos de contato foram medidos ao final dos testes de molhamento (após remoção das amostras), usando programa de análise de imagens. A interface resultante cerâmica/metal foi analisada por microscopia eletrônica de varredura. Nos resultados selecionados para brasagem de alto-vácuo $\left(<3 \times 10^{-5} \mathrm{mbar}\right)$, empregou-se o ciclo térmico padrão dado na Fig. 3. Há dois patamares de homogeneização $\left(700{ }^{\circ} \mathrm{C}\right.$ e $\left.750{ }^{\circ} \mathrm{C}\right)$ e um terceiro patamar (T-teste) especificado para uma dada temperatura em função da liga de adição nos experimentos.

Amostras foram removidas na seção transversal de juntas estanques e preparadas metalograficamente após corte em arame adiamantado, seguido as etapas de: lixamento em papel adiamantado de granas 40, 20 e $10 \mu \mathrm{m}$; polimento em suspensão de partículas de diamante $6 \mu \mathrm{me} 3 \mu \mathrm{m}$, e polimento final em suspensão de sílica coloidal $0,025 \mu \mathrm{m}$. Microscopia óptica e eletrônica de varredura usando imagem de elétrons retro-espalhados, Cambridge Stereoscan 360 equipado com técnica linescan por energia dispersiva de raios $\mathrm{X}$ e tensão $20 \mathrm{kV}$; e Jeol JSM-6390LV associado ao equipamento Thermo Scientific. Medições de microdureza Vickers foram realizadas no equipamento Shimadzu HMV-2 (carga $200 \mathrm{~g}$ e tempo $10 \mathrm{~s}$ ). Os ensaios de resistência à flexão-3p foram executados na máquina universal de ensaios EMIC DL3000 , empregando-se célula de carga $5 \mathrm{kN}$ e velocidade de avanço do cabeçote $0,5 \mathrm{~mm} / \mathrm{min}$ baseado na norma ASTM C1161-02c. Os cálculos adotados para a tensão de ruptura no 


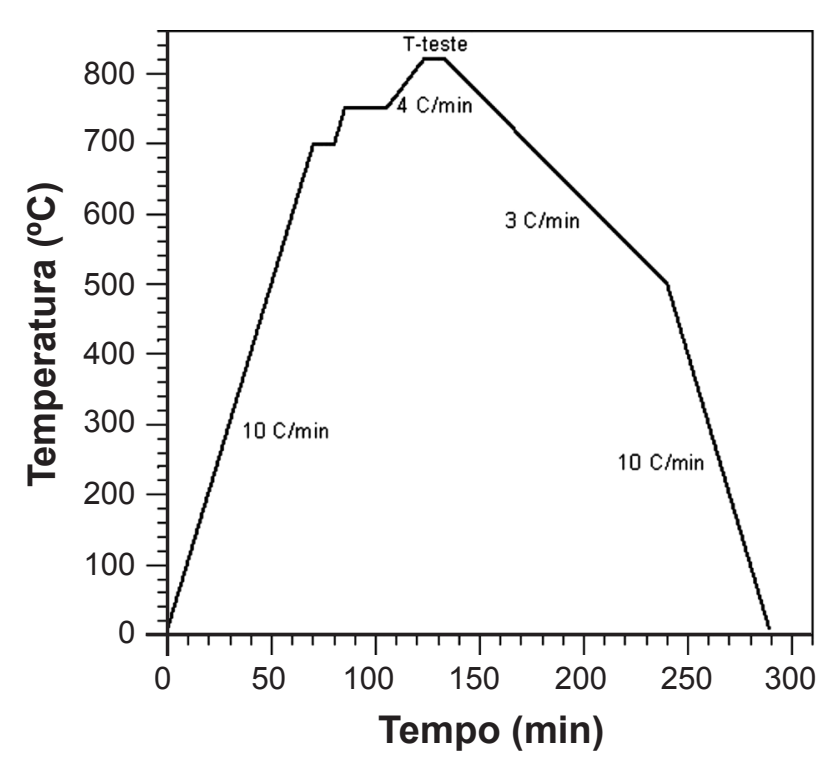

Figura 3: Ciclo térmico padrão usado nos testes de molhamento e brasagem.

[Figure 3: Standard thermal cycle used for wetting and brazing tests.]

ensaio de flexão-3p foram baseados nas equações clássicas de resistência dos materiais, estando resumidos pela Equação A (unidades S.I.): $F$ é a carga máxima atingida no ensaio; $L$ a distância do ponto de aplicação da carga ao ponto de apoio; $c$ a distância da linha neutra à superfície do corpo de prova, $\mathrm{CP}$, (neste caso, amostra cilíndrica é o valor do raio); e $J$ o momento de inércia para CPs de seção circular.

$$
\sigma_{\text {flexão - 3p }}=\frac{M_{f}}{W}=\frac{F \cdot L \cdot c}{4 J}
$$

\section{RESULTADOS E DISCUSSÃO}

$\mathrm{O}$ revestimento de $\mathrm{Ti}$ depositado mecanicamente e examinado por microscopia eletrônica apresentou espessura na faixa 5,0 $\pm 2,5 \mu \mathrm{m}$. A molhabilidade no substrato cerâmico pela liga de adição fundida foi um fator-chave na união cerâmica/metal. Os resultados dos testes de molhamento e as condições de temperatura selecionadas para brasagem indireta estão resumidos na Tabela II. A atuação do Ti como metal ativo foi eficiente para ambas as ligas, porém a melhor condição ocorreu com a liga eutética Ag-28Cu (ângulos de contato $\sim 0^{\circ}$ ). A liga Au-18Ni exibiu molhamento parcial, ou seja, houve espalhamento pobre na superfície cerâmica apesar dos ângulos de contato na faixa $40^{\circ}-45^{\circ}$ para os testes a $980{ }^{\circ} \mathrm{C} / 10 \mathrm{~min}$; a liga de adição fundida não ficou aderida à cerâmica para $1050{ }^{\circ} \mathrm{C} / 10 \mathrm{~min}$. A adição de Ti às ligas de adição $\mathrm{Ag}-\mathrm{Cu}$ resulta menores ângulos de contato $\theta$, acentuando o molhamento e adesão da liga ao substrato cerâmico [4, 9, 18]. Contradizendo ao que se encontra na literatura, onde bastaria $\theta<90^{\circ}$ para a condição de molhamento, pode-se argumentar que valores entre $70^{\circ}$ e $90^{\circ}$ seriam insuficientes para se ter um molhamento satisfatório na superfície cerâmica [14, 19].

A Fig. 4 exibe amostras significativas dos testes de molhamento com a liga de adição Ag- $28 \mathrm{Cu}$ a $820{ }^{\circ} \mathrm{C} / 10 \mathrm{~min}$ (ver Figs. 4a e 4b) e da liga Au- $18 \mathrm{Ni}$ a $980^{\circ} \mathrm{C} / 10 \mathrm{~min}$ (ver Fig. 4c). A temperatura no patamar de $820^{\circ} \mathrm{C}$ foi mais satisfatória do que $880{ }^{\circ} \mathrm{C}$ nos testes com $\mathrm{Ag}-28 \mathrm{Cu}$, pois nesta última não se formou uma fina camada de reação $(\sim 1 \mu \mathrm{m})$ junto à superfície metalizada quando observada no microscópio eletrônico de varredura e também verificada por análise EDX, a qual se mostrou composta por uma solução sólida eutética $\mathrm{Ag}-\mathrm{Cu}$ saturada em titânio. Pesquisadores já registraram que temperaturas em $\sim 820{ }^{\circ} \mathrm{C}$ podem assegurar a formação de uma contínua camada de reação na interface cerâmica/liga de adição, no caso de juntas brasadas zircônia/ Ti com ligas Ag-Cu [3, 4, 20]. Além disso, no caso da união com ligas de titânio estes valores de temperatura também estão abaixo da temperatura $\alpha \rightarrow \beta$ transus da liga Ti-6Al$4 \mathrm{~V}\left(980{ }^{\circ} \mathrm{C}\right)$, de modo a se evitar um crescimento excessivo de grãos no campo de fase- $\beta$ desta liga de titânio [2, 17].

Foi utilizado um detector de vazamento de gás hélio à

Tabela II - Resultados dos testes de molhamento e condição selecionada para brasagem.

[Table II - Results of wetting tests and selected condition for brazing temperature.]

\begin{tabular}{|c|c|c|c|c|}
\hline & \multicolumn{4}{|c|}{ Ligas de adição } \\
\hline \multirow{3}{*}{$\begin{array}{c}\text { Metal } \\
\text { ativo }\end{array}$} & Ag & & \multicolumn{2}{|c|}{ Au-18Ni } \\
\hline & \multicolumn{4}{|c|}{ Temperatura $\left({ }^{\circ} \mathrm{C}\right) /$ patamar $10 \mathrm{~min}}$. \\
\hline & 820 & 880 & 980 & 1050 \\
\hline $\mathrm{Ti}$ & \multicolumn{2}{|c|}{ molhamento } & parcial $^{\text {a }}$ & não molhou \\
\hline
\end{tabular}

${ }^{a}$ Valores $\theta<90^{\circ}$ e espalhamento pobre na cerâmica.

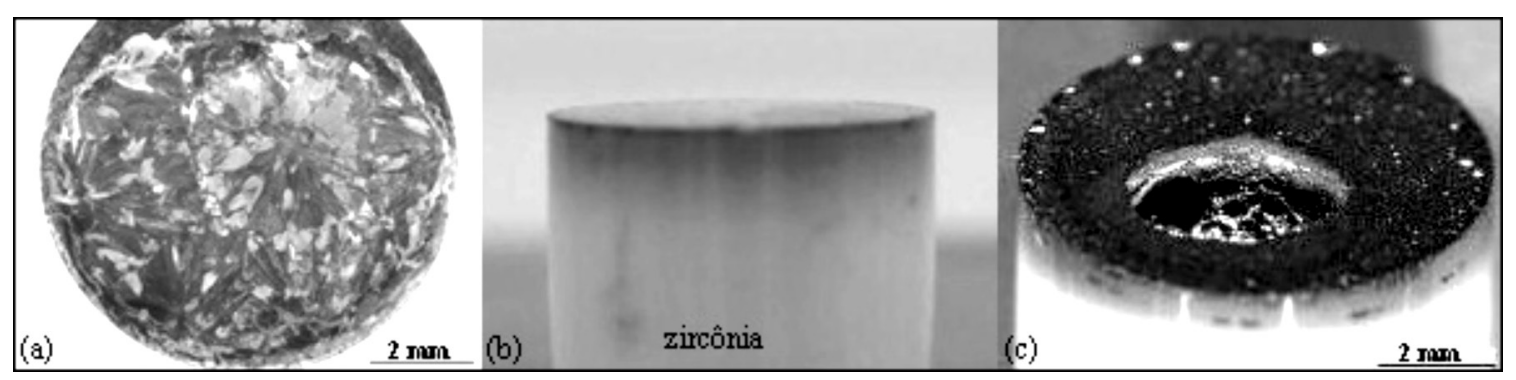

Figura 4: Testes de molhamento (a), (b) liga Ag-28Cu (820 $\left.{ }^{\circ} \mathrm{C} / 10 \mathrm{~min}\right)$; (c) liga Au-18Ni (980 $\left.{ }^{\circ} \mathrm{C} / 10 \mathrm{~min}\right)$ [12]. [Figure 4: Wetting tests (a), (b) using Ag-28Cu filler at $820^{\circ} \mathrm{C} / 10 \mathrm{~min}$; (b) using Au-18Ni filler at $980{ }^{\circ} \mathrm{C} / 10 \mathrm{~min}$ [12].] 
Tabela III - Resultados dos testes de estanqueidade nas juntas brasadas.

[Table III - Results of the He gas leak tests for brazed joints.]

\begin{tabular}{|c|c|c|}
\hline \multicolumn{3}{|c|}{ Brasagem $820^{\circ} \mathrm{C} / 10 \mathrm{~min}$, liga $\mathrm{Ag}-28 \mathrm{Cu}$} \\
\hline $\begin{array}{c}\text { Metal } \\
\text { ativo }\end{array}$ & Juntas cerâmica/metal & $\begin{array}{c}\text { Taxas de vazamento } \\
\text { (mbar.l/s) }\end{array}$ \\
\hline $\mathrm{Ti}$ & Y-TZP/Ag-28Cu/Ti-6Al-4V & $<10^{-8}$ \\
\hline \multicolumn{3}{|c|}{ Brasagem $980^{\circ} \mathrm{C} / 10 \mathrm{~min}$, liga $\mathrm{Au}-18 \mathrm{Ni}$} \\
\hline $\mathrm{T}$ & Y-TZP/Au-18Ni/Ti-6Al-4V & $10^{-3}-10^{-6}$ \\
\hline
\end{tabular}

temperatura ambiente ao redor da interface de união das juntas brasadas. Na Tabela III são dados os resultados da estanqueidade, sendo que foram consideradas satisfatórias taxas de vazamento abaixo de $10^{-8}$ mbar.l/s nas juntas Y-TZP/Ag-28Cu/Ti-6Al-4V. Entretanto, os resultados foram insatisfatórios nas juntas obtidas com a liga $\mathrm{Au}-18 \mathrm{Ni}$. Acredita-se que a taxa de vazamento admissível máxima em grande parte dos casos de aplicação técnica está na faixa de $10^{-6}-10^{-8} \mathrm{mbar} .1 / \mathrm{s}$ e que, especificamente, valores registrados para taxas abaixo de $10^{-8} \mathrm{mbar} .1 / \mathrm{s}$ permitem classificar um componente como estanque. Por exemplo, neste último significa que haveria perdas de $3 \mathrm{~mL}$ de gás hélio em 1 ano através de uma descontinuidade com diâmetro $\sim 0,4 \mu \mathrm{m}$. No caso da indústria automotiva o limite superior para taxas de vazamento entre $10^{-4}$ e $10^{-6} \mathrm{mbar} .1 / \mathrm{s}$ possibilita classificar um componente como estanque a gasolina (inclusive vapor) vazamentos na ordem de $10^{-6} \mathrm{mbar} .1 / \mathrm{s}$ significam perdas de $\sim 1 \mathrm{~cm}^{3}$ de gás hélio em 12 dias [21].

\section{Escurecimento na zircônia}

A Fig. 5 apresenta o efeito de escurecimento ocorrido na zircônia junto à interface cerâmica/metal durante o ciclo de brasagem. Este se mostrou relativamente mais pronunciado quando utilizada a liga $\mathrm{Au}-18 \mathrm{Ni}$ em relação aos resultados verificados com a liga $\mathrm{Ag}-28 \mathrm{Cu}$. Vale ressaltar que, as temperaturas de união foram apreciavelmente distintas: 980 ${ }^{\circ} \mathrm{C} / 10$ min para a primeira, $820{ }^{\circ} \mathrm{C}$ e $880{ }^{\circ} \mathrm{C} / 10$ min para a segunda liga de adição. Pesquisadores têm registrado este típico escurecimento na zircônia para uniões aos metais na brasagem com ligas de adição Ag-Cu ativas (presença de \% Ti na liga) $[2,3,8,9]$. Este fenômeno pode ser atribuído a uma remoção parcial de íons oxigênio pelos cátions do metal ativo (Ti), criando-se uma área deficiente em oxigênio na porção cerâmica. Em outras palavras, formouse uma zircônia não-estequiométrica na região escurecida com déficit em oxigênio $\left(\mathrm{ZrO}_{2-\mathrm{x}}, 0<X \leq 0,02\right)$ pelo enfraquecimento das ligações $\mathrm{Zr}-\mathrm{O}$. Consequientemente, a condição de molhamento pela liga fundida é favorecida na superfície cerâmica devido à reação de redução do óxido de zircônio ocasionada pela alta afinidade química do elemento titânio pelo oxigênio, resultando na formação de ligações químicas adequadas para união da zircônia ao par metálico da junta. O efeito de escurecimento da zircônia possui influência direta de alguns fatores, tais como: temperatura e tempo de patamar na brasagem; material usado como par metálico; e o percentual de metal ativo na composição da liga de adição [2, 3]. Pode-se incluir também a influência do tipo de estrutura cerâmica: a zircônia monocristalina apresenta escurecimento mais acentuado do que o verificado na zircônia policristalina, por exemplo [22].

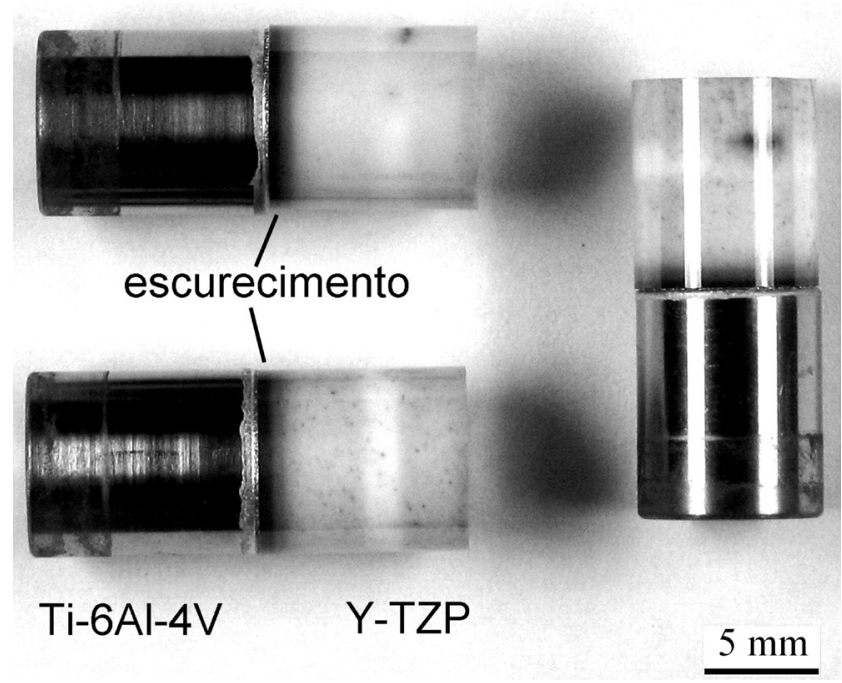

Figura 5: Escurecimento na zircônia (brasagem com a liga Ag$\left.28 \mathrm{Cu}, 820^{\circ} \mathrm{C} / 10 \mathrm{~min}\right)$.

[Figure 5: Darkening effect in zirconia (brazing with $\mathrm{Ag}-28 \mathrm{Cu}$ filler, $\left.\left.820^{\circ} \mathrm{C} / 10 \mathrm{~min}\right).\right]$

Cabe salientar que, conforme indicado no diagrama de Ellingham, o composto $\mathrm{ZrO}_{2}$ é mais estável do que os conhecidos óxidos de titânio, ou seja, a redução química da zircônia pelo titânio é termodinamicamente desfavorável. Admite-se, então, que as reações de redução nesta cerâmica óxida possam ocorrer no nível de algumas escalas atômicas a partir da superfície, uma vez que o valor de $\Delta G$ na superfície do material tende a ser considerado menor em valor absoluto - $\Delta G$ é maior (valor menos negativo), possivelmente, influenciado pelo maior grau de liberdade de vibração dos átomos, diferentemente da condição encontrada no interior do material [23]. Quanto à redução ocorrida na superfície da zircônia, deve-se notar que os valores $\Delta G(\mathrm{em} \mathrm{kJ} / \mathrm{mol})$ para formação dos compostos $\mathrm{TiO}$ e $\mathrm{ZrO}_{2}$ (-867,37 e -891,25, respectivamente) estão muito próximos. Então, considerando a afirmação anterior, é provável que o valor de $\Delta G$ na superfície da cerâmica seja relativamente superior àquele para a formação dos óxidos de titânio, fato que viabilizaria a redução química da zircônia pela ação deste elemento [12].

\section{Propriedades mecânicas das juntas brasadas}

Na Fig. 6 são mostrados perfis de microdureza através das juntas Y-TZP/Ag-28Cu/Ti-6Al-4V (brasagem no patamar $\left.820{ }^{\circ} \mathrm{C} / 10 \mathrm{~min}\right)$, com indentações Vickers $\left(\mathrm{HV}_{0,2}\right)$ em $\sim 50 \mu \mathrm{m}$ a partir da interface de união. A simbologia (Ti) no gráfico significa metalização prévia com titânio na cerâmica. Notou-se que a dureza foi pouco inferior dentro 
da região de brasagem em comparação aos valores médios verificados no metal base (Ti-6Al-4V). Entenda-se aqui por "região de brasagem" a extensão da interface cerâmica/ metal, sobretudo na contraparte metálica da união, visível à luz da microscopia óptica para as medições. Provavelmente houve alguma difusão de prata e cobre da liga de adição para o metal base, contribuindo na formação de intermetálicos nesta leve redução na dureza notada na região brasada. Os valores medidos aumentaram rapidamente ao atingir o material cerâmico mostrando acentuada descontinuidade na interface. As medições de dureza feitas a partir da interface de união para o interior da cerâmica não indicaram uma alteração expressiva entre as mesmas, apesar do efeito de escurecimento ocorrido na zircônia (vide Fig. 5).

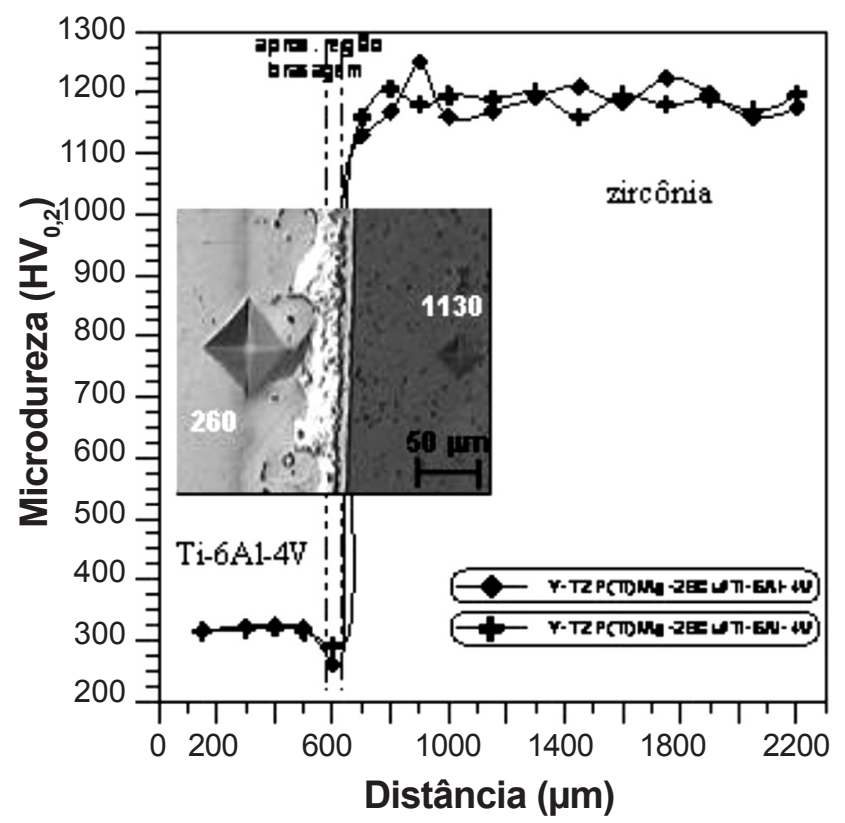

Figura 6: Perfis de microdureza através da interface de juntas Y-TZP/Ag-28Cu/Ti-6Al-4V.

[Figure 6: Microhardness profiles across the brazed joints interface Y-TZP/Ag-28Cu/Ti-6Al-4V.]

Os resultados do ensaio de flexão-3p nas juntas brasadas são dados na Tabela IV. A resistência mecânica obtida para as uniões com a liga $\mathrm{Ag}-28 \mathrm{Cu}$ foi muito superior àquela verificada com a liga $\mathrm{Au}-18 \mathrm{Ni}$, haja vista que as taxas de vazamento foram superiores neste último grupo (vide Tabela III). Adispersão dos resultados relativamente baixa no primeiro grupo de juntas pode ser atribuída a uma concentração de tensões não elevada na interface de união, indicando também uma boa condição de reprodutibilidade quanto ao processo de brasagem. Além disso, pode-se dizer que a resistência mecânica de juntas brasadas e a formação da área escura deficiente em oxigênio na zircônia antes mencionada são independentes entre si, ou seja, não se constatou um efeito negativo mensurável nesta situação $[2,3]$.

A possibilidade de formação de tensões residuais na interface de juntas cerâmica/metal, quando em função dos diferenciados coeficientes de expansão térmica linear dos componentes, pode ser vista desta forma: o valor de $\alpha$-CTE da liga Ti-6Al-4V $\left(9,7 \times 10^{-6} /{ }^{\circ} \mathrm{C}\right)$ é comparável ao da zircônia $\left(10,0-10,4 \times 10^{-6} /{ }^{\circ} \mathrm{C}\right)$, mas o valor deste coeficiente é comparativamente inferior em comparação às ligas de adição - $\alpha$-CTE da liga Ag-28Cu $\left(20-400{ }^{\circ} \mathrm{C}\right)$ e da liga Au-18Ni $\left(20-550{ }^{\circ} \mathrm{C}\right.$ ) são $17,8 \times 10^{-6} /{ }^{\circ} \mathrm{C}$ e $14,6 \times 10^{-6} /{ }^{\circ} \mathrm{C}$, respectivamente. Logo, níveis de tensões residuais podem ter se originado na interface $\mathrm{ZrO}_{2} / \mathrm{Ti}-6 \mathrm{Al}-4 \mathrm{~V}$ devido às contrações diferenciadas dos componentes no resfriamento a partir da temperatura de brasagem. Contudo, considera-se que grande parte das tensões pode ser acomodada por deformação plástica em função da grande ductilidade destas ligas de adição $[2,5]$.

Tabela IV - Resultados do ensaio de flexão-3p nas juntas brasadas.

[Table IV - Results of the three-point flexure test in the brazed joints.]

\begin{tabular}{|c|c|c|}
\hline \multicolumn{3}{|c|}{ Brasagem $820^{\circ} \mathrm{C} / 10 \mathrm{~min}$, liga $\mathrm{Ag}-28 \mathrm{Cu}$} \\
\hline $\begin{array}{c}\text { Metal } \\
\text { ativo }\end{array}$ & \multirow{2}{*}{$\begin{array}{c}\text { Juntas cerâmica/metal } \\
\text { Y-TZP(Ti)/Ag-28Cu/ } \\
\text { Ti-6Al-4V }\end{array}$} & $\begin{array}{l}\text { Resistência à flexão } \\
(\mathrm{MPa})\end{array}$ \\
\hline $\mathrm{Ti}$ & & $121,7 \pm 13,4$ \\
\hline \multicolumn{3}{|c|}{ Brasagem $980^{\circ} \mathrm{C} / 10 \mathrm{~min}$, liga $\mathrm{Au}-18 \mathrm{Ni}$} \\
\hline $\begin{array}{l}\text { Metal } \\
\text { ativo }\end{array}$ & Juntas cerâmica/metal & $\begin{array}{c}\text { Resistência à flexão } \\
(\mathrm{MPa})\end{array}$ \\
\hline Ti & $\begin{array}{c}\text { Y-TZP(Ti)/Au-18Ni/Ti- } \\
6 \mathrm{Al}-4 \mathrm{~V}\end{array}$ & $62,4 \pm 23,6$ \\
\hline
\end{tabular}

Nota: cinco pares brasados foram testados em cada condição de ensaio.

Os valores da resistência à flexão-3p nas juntas brasadas com a liga $\mathrm{Ag}-28 \mathrm{Cu}$ foram satisfatórios em comparação aos resultados encontrados na literatura científica considerando-se o tipo de materiais unidos, ciclo térmico e variáveis similares no procedimento de ensaio. Foi obtida uma resistência na faixa de $246 \pm 24 \mathrm{MPa}$, usando uma liga de adição $\mathrm{Ag}$-Cu-Ti na brasagem direta de juntas $3 Y-P S Z / T i(\phi 8 \times 35 \mathrm{~mm})$ no patamar $850{ }^{\circ} \mathrm{C} / 5 \mathrm{~min}$ [2]. A força média aplicada nos ensaios de flexão do presente estudo, possivelmente, foi da mesma ordem de grandeza no estudo em [2]. Além disso, foi utilizada igual velocidade de avanço no carregamento contra uma seção resistente similar. Devido à geometria das juntas, pode-se supor que aqueles pesquisadores utilizaram $\mathrm{L}=20 \mathrm{~mm}$ na execução dos ensaios de flexão (distância entre apoios inferiores) valor desta variável indicado na configuração mínima do ensaio, segundo norma ASTM C1161-02c. No presente estudo foi usado $\mathrm{L}=10 \mathrm{~mm}$ (valor sub-dimensionado). Para fins comparativos a tensão de ruptura nas juntas Y-TZP/Ag-28Cu/Ti-6Al-4V foi $122 \pm 13 \mathrm{MPa}$ (ver Tabela IV) e, portanto, tal valor seria equivalente àquele registrado na literatura caso fosse usado o valor em dobro na variável L no cálculo da tensão $(122$ × $2=244 \mathrm{MPa}$, vide Equação A), sendo mantidas as considerações sobre magnitude da força e configuração da junta.

Na Fig. 7 é visto um par de superfícies de fratura representativo para os ensaios de flexão-3p nas juntas Y-TZP/ 
Ag-28Cu/Ti-6Al-4V, mostrando um aspecto côncavoconchoidal após a ruptura. Houve arrancamento severo de material cerâmico, permanecendo este totalmente aderido junto ao par metálico. Fica sugerido que o trincamento se iniciou na região externa da interface cerâmica/liga de adição, propagando-se bruscamente para o interior da união. Tal fato evidenciou uma associação satisfatória entre a liga fundida e o revestimento de metal ativo, resultando em adequadas ligações químicas para a união cerâmica/metal.

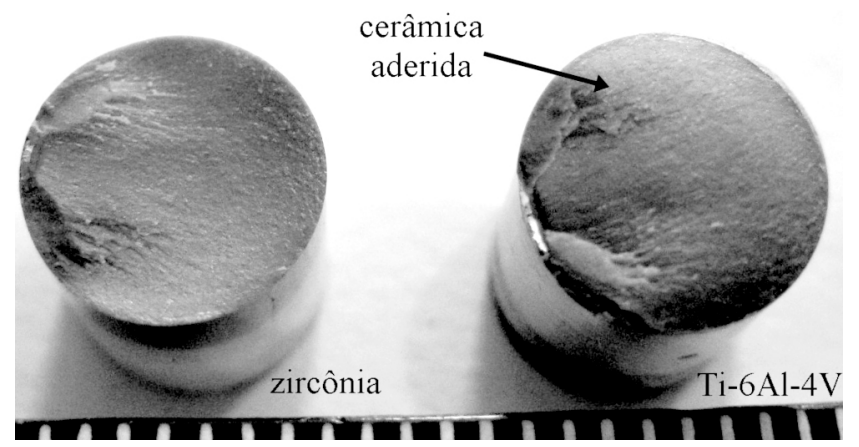

Figura 7: Superfície de fratura da junta Y-TZP/Ag-28Cu/Ti-6Al4V testada por flexão-3p. Dimensão mm.

[Figure 7: Fracture surface of the joint Y-TZP/Ag-28Cu/Ti-6Al-4V submitted for three-point flexure test. Dimension in $\mathrm{mm}$.]

\section{Análise microestrutural}

As imagens de microscopia eletrônica de varredura na Fig. 8 representam a interface resultante nas juntas Y-TZP/Ag$28 \mathrm{Cu} / \mathrm{Ti}-6 \mathrm{Al}-4 \mathrm{~V}$ brasadas no patamar $820^{\circ} \mathrm{C} / 10 \mathrm{~min}$. A Fig. 8a sugere a formação de uma interface confiável, destacandose três regiões distintas: camada escura adjacente à cerâmica metalizada com Ti; camada intermetálica na interface liga fundida/metal base; e uma zona difusiva no par metálico da união. Análises EDX feitas na região de brasagem indicaram na faixa clara maiores níveis do elemento $\mathrm{Ag}$ e na faixa cinzenta para $\mathrm{Cu}$. Na Fig. $8 \mathrm{~b}$ aplicouse a técnica linescan (análise em linha por dispersão de energia de raios $\mathrm{X}$ ) através da interface zona difusiva/liga de adição. Houve alguma difusão dos elementos $\mathrm{Cu}$ e $\mathrm{Ag}$ para formação da zona difusiva, inclusive constatou-se a difusão de Ti do par metálico para o interior da liga fundida. A análise elementar quantitativa (em percentual-atômico) indicou na zona difusiva: $\mathrm{Ti}(\sim 77 \%$ at.), $\mathrm{Cu}(\sim 5 \%$ at.), $\mathrm{Ag}$ ( $1 \%$ at.) e outros (Al, V, balanço). Esta camada intermetálica (vista na Fig. 8b) é uma região rica em $\mathrm{Ti}(\sim 73 \%$ at.) e $\mathrm{Cu}$ ( $\sim 18 \%$ at.), contendo baixos percentuais de $\mathrm{Ag}, \mathrm{Al}$ e V. Sabese que a prata possui baixa difusividade através da camada $\mathrm{CuTi}$, apesar de sua boa solubilidade em titânio e capacidade de formar intermetálicos com tal elemento $\left(\mathrm{AgTi}_{2}\right.$, p. ex.); além disso, o elemento cobre possui forte tendência para uma rápida formação de intermetálicos com titânio [2, 3] Baseado nestes resultados e após consultas ao diagrama de equilíbrio $\mathrm{Cu}-\mathrm{Ti}$, fica sugerida uma provável formação do composto $\mathrm{CuTi}_{2}$ nas regiões citadas anteriormente. $\mathrm{Na}$ literatura científica, após a revisão do diagrama de equilíbrio $\mathrm{Cu}-\mathrm{Ti}$ ficaram definidas as regiões de cinco compostos intermetálicos: $\beta-\mathrm{Cu}_{4} \mathrm{Ti}, \mathrm{Cu}_{2} \mathrm{Ti}_{1} \mathrm{Cu}_{3} \mathrm{Ti}_{2}, \mathrm{CuTi}$ e $\mathrm{CuTi}_{2}$ [24]. Pesquisadores já registraram que na brasagem direta de juntas zirconia/Ti com uso de ligas ativas houve a formação dos compostos CuTi e $\mathrm{CuTi}_{2}$ na interface resultante da zona difusiva/liga fundida $[2,3,19]$.

A Fig. 9 exibe imagens MEV destacando a interface cerâmica/liga de adição, referente àquela imagem da Fig. 8 . Na Fig. 9a tem-se uma camada escura $(\sim 1,5 \mu \mathrm{m})$ adjacente à superfície cerâmica metalizada, além de uma zona de precipitação formada entre esta e a liga fundida. A aplicação da técnica linescan através desta interface ratificou que houve difusão de Ti do par metálico da junta para o interior da liga fundida, bem como a partir da superfície metalizada adentrando no material cerâmico (profundidade inferior
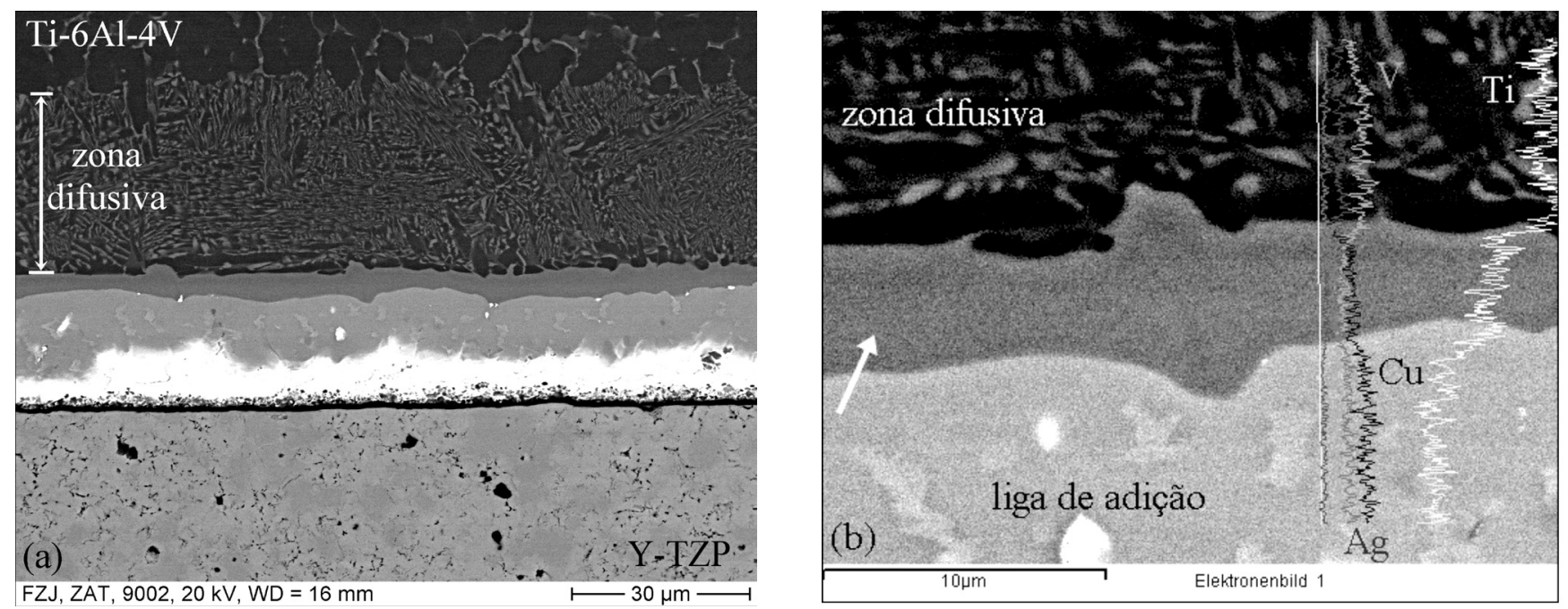

Figura 8: Imagens MEV - (a) interface Y-TZP/Ag-28Cu/Ti-6Al-4V, camada escura adjacente à superfície cerâmica metalizada; (b) técnica linescan através da camada intermetálica (ver seta branca).

[Figure 8: SEM images-(a) Y-TZP/Ag-28Cu/Ti-6Al-4V joint interface, dark layer next to the metallized ceramic surface; (b) linescan technique through the intermetallic layer (see white arrow).] 
a $5 \mu \mathrm{m})$. A análise elementar quantitativa (análise EDX) nesta camada escura resultou: Ti ( $\sim 57 \%$ at.), O ( 39\% at.) e nos elementos $\mathrm{Zr}$, Ag e $\mathrm{Cu}$ (balanço). Portanto, trata-se de uma região rica em $\mathrm{Ti}$ e $\mathrm{O}$ contendo provavelmente compostos $\mathrm{TiO}_{\mathrm{x}}$ a partir da redução do óxido de zircônio pelo titânio. Cabe ressaltar que, esta camada escura adjacente à superfície cerâmica metalizada não é aquela região resultante do efeito de escurecimento anteriormente comentado no presente estudo. A primeira está inserida em nível microscópico (primeiros planos atômicos) dentro da região de escurecimento da zircônia. Estudos registraram que esta camada escura de reação resultou da brasagem direta da zircônia a metais quando usada uma liga Ag- $\mathrm{Cu}$ contendo Ti, sendo tal camada basicamente composta por óxidos do tipo TiO necessários para favorecer o molhamento na cerâmica $[4,9,18,19]$. O composto TiO é o mais freqüente óxido de titânio formado na redução superficial da zircônia frente aos demais óxidos resultantes pela ação deste metal ativo (TiO, $\mathrm{Ti}_{2} \mathrm{O}_{3}, \mathrm{Ti}_{3} \mathrm{O}_{5} \ldots \mathrm{TiO}_{2}$ ) [2-4, 22]. Na Fig. 9b é mostrada uma região de descontinuidade na camada escura presente na superfície cerâmica, onde provavelmente houve deposição insuficiente de Ti durante a etapa de metalização mecânica. Aplicando-se a técnica linescan nesta chamada "área de defeito" foram detectados níveis EDX para os elementos Ti, $\mathrm{Ag}, \mathrm{Cu}$ e $\mathrm{Zr}$ (este pela redução do composto $\mathrm{ZrO}_{2}$ ), ou seja, a liga de adição tornou-se ativa in situ. Embora houvesse menor disponibilidade local de Ti nestas áreas, a liga fundida tornou-se ativa na temperatura de brasagem. Camadas de reação em superfícies cerâmicas metalizadas mecanicamente costumam apresentar alguma descontinuidade, pelo fato de antes já ter sido formado um revestimento metálico de aspecto sinuoso e descontínuo $[14,16]$.

A Fig. 10 mostra imagens $\mathrm{MEV}$ na interface da junta Y-TZP/Au-18Ni/Ti-6Al-4V. Nota-se que há uma zona
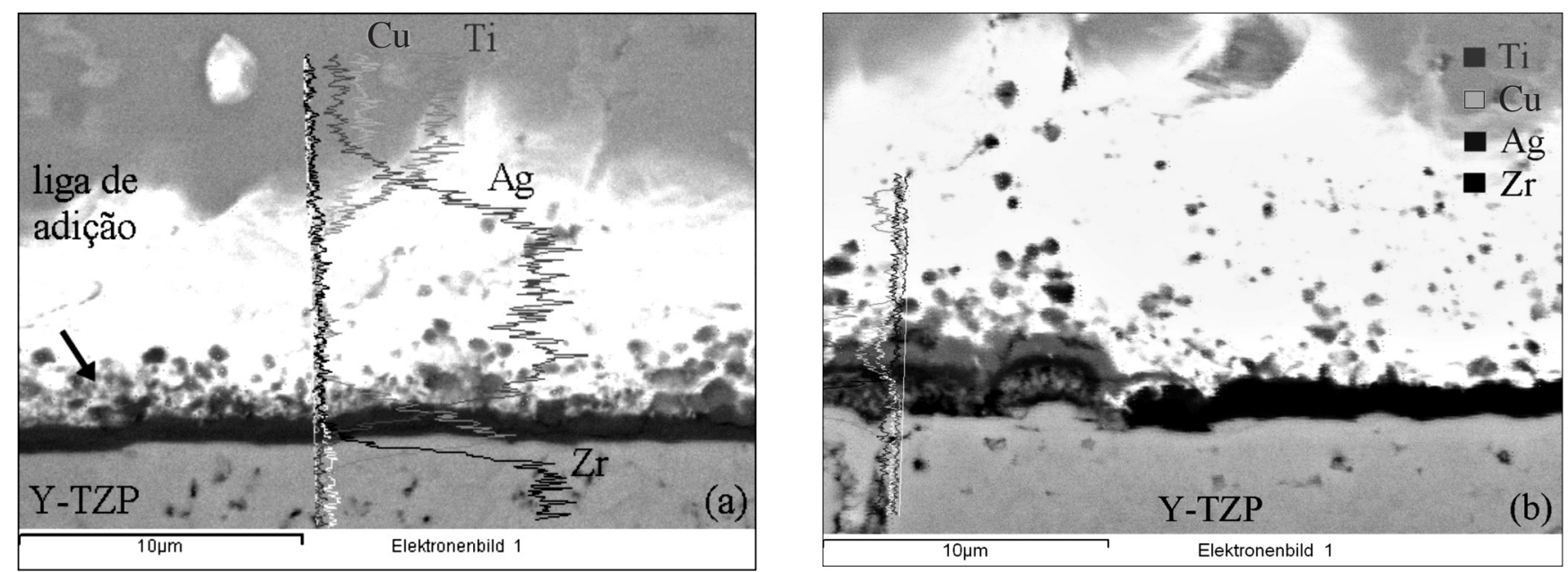

Figura 9: Imagens MEV - (a) técnica linescan através da interface Y-TZP/liga de adição, zona de precipitação junto à camada escura na superfície cerâmica (ver seta); (b) técnica linescan numa região de descontinuidade.

[Figure 9: SEM images - (a) linescan technique through the Y-TZP/filler interface, precipitation zone next to the dark layer on the ceramic surface (see arrow); (b) linescan technique through the discontinued region.]
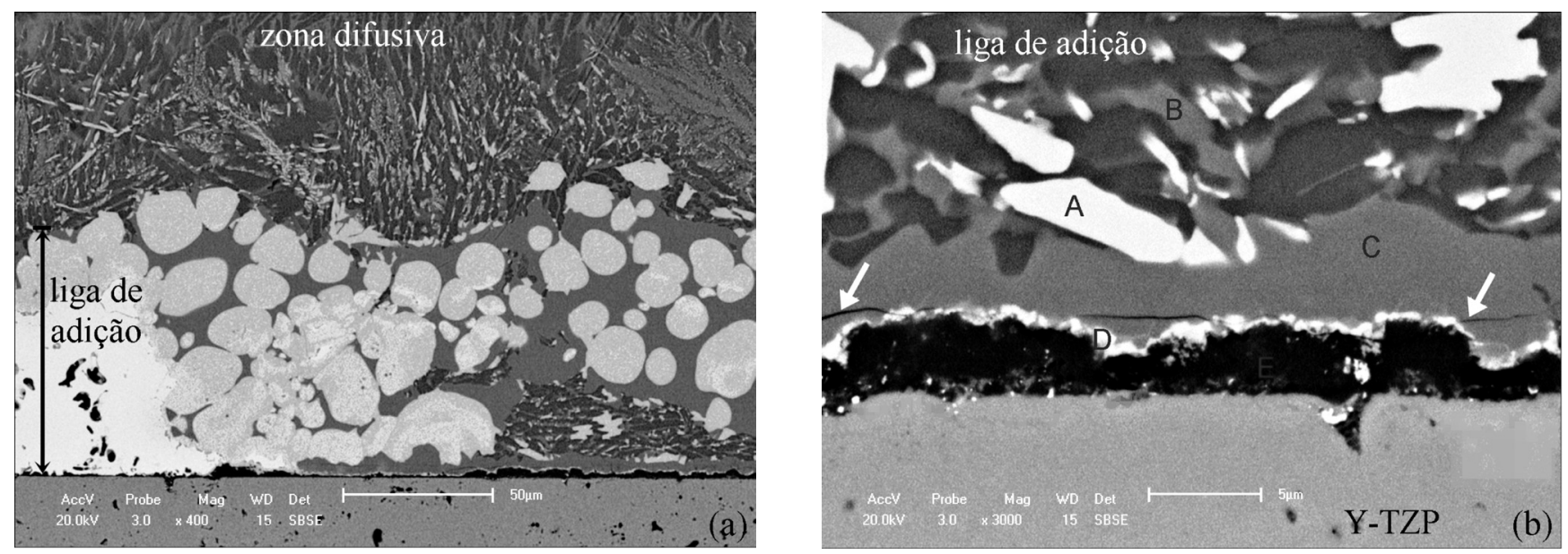

Figura 10: Imagens MEV - (a) interface Y-TZP/Au-18Ni/Ti-6Al-4V; (b) Análise EDX pontual (ver letras indicativas), presença de intermetálicos e microtrincamento (ver seta branca).

[Figure 10: SEM images - (a) Y-TZP/Au-18Ni/Ti-6Al-4V joint interface; (b) EDX analysis (see indicative points), presence of intermetallics and microcracking (see white arrow).] 
difusiva formada no par metálico da união, além de uma diferenciada precipitação de fases intermetálicas na região de brasagem (ver Fig. 10a). A análise EDX via técnica linescan através da interface zona difusiva/liga de adição constatou que houve difusão dos elementos $\mathrm{Au}$ e $\mathrm{Ni}$ da liga fundida para o contato no par metálico. Verificou-se haver microtrincamento ao longo da interface cerâmica/liga de adição (ver Fig. 10b). A excessiva presença de intermetálicos provavelmente gerou altas tensões residuais na rede cristalina da região de brasagem, favorecendo a ocorrência do trincamento que também pode estar associado às diferenças entre os valores $\alpha$-CTE dos membros individuais da junta. Algumas fases intermetálicas foram caracterizadas via análise EDX, possivelmente para os seguintes compostos: Ti-Au, Ti-Al-Ni-V e Ti-Al-Ni respectivamente nas regiões A, B e C indicadas na Fig. 10b. Sabe-se que o sistema binário $\mathrm{Au}$-Ti possui uma série de compostos bem definidos, tais como: $\mathrm{Ti}_{3} \mathrm{Au}, \mathrm{TiAu}_{2}, \mathrm{TiAu}_{4}$ e alternando-se $\alpha, \beta$ na fase $\gamma$-TiAu [25]. A região D indicada na Fig. 10b é uma camada de reação muito fina $(0,5-1,0 \mu \mathrm{m})$, que contém os elementos $\mathrm{Ti}$, Au e Ni. Na região E (camada escura) foram obtidos altos níveis EDX para Ti e menores para $\mathrm{Zr}$ - o primeiro devido à técnica de metalização, e o segundo pela redução do óxido de zircônio. Considerou-se que o titânio do revestimento reagiu em grande parte com o ouro e níquel na intensa precipitação de intermetálicos e, então, não permanecendo este metal ativo em solução sólida com atividade suficiente para uma adequada redução química do composto $\mathrm{ZrO}_{2}$ (principal constituinte da zircônia) na superfície cerâmica.

\section{CONCLUSÕES}

A técnica de metalização mecânica demonstrou ser viável para obtenção de juntas estanques Y-TZP/Ti-6Al-4V pelo processo de brasagem indireta. As juntas brasadas com a liga eutética $\mathrm{Ag}-28 \mathrm{Cu}$ apresentaram resistência mecânica à flexão$3 p$ satisfatória. Formou-se uma camada escura adjacente à superfície cerâmica metalizada e responsável pelo bom molhamento da mesma, além de uma camada intermetálica (rica em Cu-Ti) na interface Ag-28Cu/Ti-6Al-4V. A presença excessiva de intermetálicos na região de brasagem para uniões obtidas com a liga Au-18Ni pode ter causado distorções na rede cristalina formada nesta região, ocasionando microtrincamento interfacial - haja vista as maiores taxas de vazamento detectadas nesta condição. O titânio possivelmente reagiu em grande parte com ouro e níquel na precipitação de intermetálicos, não ficando em solução sólida com atividade suficiente para redução da zircônia.

\section{AGRADECIMENTOS}

Ao corpo técnico do FZ-Juelich pela parceria e assistência tecnológica, além do apoio prestado nos ensaios mecânicos e análises de microscopia eletrônica nos laboratórios GRANTE e LCME da UFSC, respectivamente. J. S. Pimenta agradece ao projeto PROBRAL Capes/BR-DAAD, por patrocinar esta pesquisa de cooperação e à agência $\mathrm{CNPq}$ pelo oferecimento de sua bolsa de doutorado no Brasil.

\section{REFERÊNCIAS}

[1] G. W. Liu, W. Li, G. J. Qiao, H. J. Wang, J. F. Yang, T. J. Lu, J. Alloys Compd. 470 (2009) 163.

[2] Q. Smorygo, J. S. Kim, M. D. Kim, T. G. Eom, Mater. Lett. 61 (2007) 613.

[3] W. B. Hanson, K. I. Ironside, J. A. Fernie, Acta Mater. 48 (2000) 4673.

[4] D. Sciti, A. Bellosi, L. Espósito, J. Eur. Ceram. Soc. 21 (2001) 45 .

[5] M. Singh, T. P. Shpargel, R. Asthana, J. Mater. Sci. 43, 1 (2008) 23.

[6] J. R. Kelly, I. Denry, Dental Mater. 24 (2008) 289.

[7] N. Yoshikawa, A. Kikuchi, S. Taniguchi, J. Mater. Sci. 34 (1999) 5885.

[8] M. Singh, T. P. Shpargel, R. Asthana, Int. J. Appl. Ceram. Technol. 4, 2 (2007) 119.

[9] M. L. Muolo, E. Ferrera, L. Morbelli, Scripta Mater. 50 (2004) 325.

[10] A. K. Jadoon, J. Mater. Sci. 39 (2004) 593.

[11] R. M. Nascimento, "Metalização mecânica de $\mathrm{Al}_{2} \mathrm{O}_{3}$ para brasagem metal-cerâmica" Tese Dr., Faculdade de Engenharia Mecânica, Universidade Federal de Santa Catarina, Florianópolis, SC (2001) 135p.

[12] J. S. Pimenta, "Metalização mecânica para brasagem de uniões $\mathrm{ZrO}_{2} /$ metal e $\mathrm{ZrO}_{2} / \mathrm{ZrO}_{2}$ ", Tese Dr., Faculdade de Engenharia Mecânica, Universidade Federal de Santa Catarina, Florianópolis, SC (2010) 233p.

[13] F. Löffler, Surface \& Coatings Technol. 132 (2000) 222.

[14] R. M. Nascimento, A. E. Martinelli, A. J. A. Buschinelli, Cerâmica 49, 312 (2003) 178.

[15] G. Elssner, G. Petzow, ISIJ Int. 30, 2 (1990) 1011.

[16] R. M. Nascimento, A. J. A. Buschinelli, A. E. Martinelli, E. Sigismund, J. Remmel, U. Reisgen, E. Lugscheider, in $6^{\text {th }}$ Int. Conf. Joining Ceramic, Glass and Metal, Munich, Alemanha (2002) 58.

[17] ASM Material Data Sheet, http://asm.matweb.com, acessado 25/05/2009.

[18] K. S. Weil, J. Y. Kim, J. S. Hardy, J. T. Darsell, Scripta Mater. 54 (2006) 1071.

[19] O. M. Akselsen, J. Mater. Sci. 27 (1992) 1989.

[20] H. Q. Hao, Y. L. Wang, Z. H. Jin, X. T. Wang, J. Mater. Proc. Technol. 52 (1995) 238.

[21] G. Schröder, J. C. P. Filho, W. Schmidt, Fundamentos de Ensaio de Vazamento e Estanqueidade, Reihe Allgemeines/ General, Band 1 - Forschungszentrum Jülich GmbH, Jülich, Alemanha (2000) 38.

[22] A. V. Durov, Y. V. Naidich, B. D. Kostyuk, J. Mater. Sci. 40 (2005) 2173.

[23] P. R. Chidambaram, G. R. Edwards, D. L. Olson, Met. Trans. B, 23(B) (1992) 215.

[24] H. Okamoto, J. Phase Equil. 26, 3 (2002) 549.

[25] W. Luo, Z. Jin, H. Liu, T. Wang, Calphad 25, 1 (2001) 19. (Rec. 21/06/2011, Ac. 21/07/2011) 